Jpn. J. Health Phys., 40(1)，79-86 (2005)

\title{
Report
}

\section{Report on the Status of Regulation for NORM/TENORM in Australia}

\author{
Lubi DIMITROVSKI ${ }^{* 1}$
}

(Received on March 2, 2005)

\begin{abstract}
Australia is a major producer and exporter of ores and minerals (coal, oil, gas, iron ore, bauxite, phosphate rock and mineral sands) which unfortunately can contain NORM. In some industries the management of possible NORM/TENORM exposures is already being. In the oil and gas industry for example radiation protection measures are implemented for staff undertaking maintenance on non-contaminated equipment. However there is still widespread non-regulation in other industries that adequately address the potential occupational and public health concerns relating to NORM/TENORM. The application of radiological protection regulations is currently not uniform throughout Australia. There are nine separate jurisdictions (State, Territory and Commonwealth) having responsibility for radiation safety legislation associated with artificial and naturally occurring radioactive material (NORM). The lack of uniformity in areas such as licensing, exemption limits and the fact that some parts of regulations in individual jurisdictions do not meet current international best practice, creates problems across jurisdictions.
\end{abstract}

KEY WORDS: NORM, TENORM, regulation, radiation safety, exemption, ARPANSA, concentration, transportation, uniformity, transport.

\section{INTRODUCTION}

This report outlines Australia's inventory of technologically enhanced naturally occurring radioactive material, current regulations, and further identifies a number of issues to be solved in the future.

Naturally occurring radioactive material (NORM) is distributed throughout the earth's crust and contains nuclides from the ${ }^{238} \mathrm{U},{ }^{235} \mathrm{U}$ and ${ }^{232} \mathrm{Th}$ decay series, as well as other longlived radionuclides such as ${ }^{40} \mathrm{~K}$. These nuclides give rise to "background" radiation, which varies by two orders of magnitude over the earth's surface.

The widespread occurrence of NORM means that many of the ores and minerals (coal, oil and gas, iron ore, bauxite, phosphate rock), commodities (water, building materials, fertiliser), products (ceramics), and other devices (welding rods, gas mantles and electronic components) used by humans can contain NORM. Activities such as mineral processing, coal burning (for electricity generation) and water treatment can modify the NORM concentrations in the products, by-products and wastes generated by these activities. In some situations, specific radionuclides can become separated from the original radionuclide mixture, eg volatilisation of polonium

\footnotetext{
${ }^{* 1}$ Australian Nuclear Science \& Technology Organisation.
}

and lead isotopes when coal is burnt to generate electricity and the separation of radium and uranium during the processing of gypsum to produce fertiliser. When the NORM concentrations have been modified in the material, it is called technologically enhanced naturally occurring radioactive material, or TENORM.

Human health is not affected in the majority of these situations, as the activity arising from the NORM levels is not very high. However, when NORM has been significantly concentrated through large-scale industrial production, occupational and public exposure to radiation can become an issue. In some industries this is already being addressed, but in others NORM has not been recognised as a potentially significant problem.

Current and historical options for disposing of NORM wastes include landfill, near-surface disposal, land contouring and disposal into mine tailings dams. Other options include dilution in industrial waste disposal facilities, land farming by ploughing in over a gazetted disposal area, and incorporation into concrete for building construction or road base. In some cases, a lack of awareness of NORM issues in the past has led to the creation of contaminated sites for which no individual or organisation is legally accountable. The remediation of these sites will require careful consideration. 
Table 1 Summary of NORM in Australian Industries and Materials.

\begin{tabular}{|c|c|c|c|}
\hline $\begin{array}{l}\text { Category of NORM } \\
\text { I. Raw material } \\
\text { II. Product(s) } \\
\text { III. Waste/by-product }\end{array}$ & $\begin{array}{l}\text { Scale of mining/production and } \\
\text { waste generation in Australia } \\
\text { (estimated) }\end{array}$ & $\begin{array}{l}\text { Typical radionuclide concentra- } \\
\text { tions } \\
\left(\mathrm{kBq} \cdot \mathrm{kg}^{-1}\right)\end{array}$ & $\begin{array}{l}\text { Waste management or by-prod- } \\
\text { uct use } \\
\text { Mineral sand mining and pro- } \\
\text { cessing }\end{array}$ \\
\hline $\begin{array}{l}\text { Mineral sand mining and pro- } \\
\text { cessing } \\
\text { I. Ore } \\
\text { II. Heavy minerals } \\
\text { III. } \\
\text { a. Tails from primary sepera- } \\
\text { tion } \\
\text { b. Oversize from secondary } \\
\text { sepn } \\
\text { c. Tails from secondary sepn. } \\
\text { d. Dust from secondary sepn. } \\
\text { e. Solids from synthetic rutile } \\
\text { f. Kiln solids }\end{array}$ & $\begin{array}{l}\text { I. } 3.5 \mathrm{Mt} \cdot \mathrm{a}^{-1} \\
\text { II. } \\
\text { a. } 2.5 \mathrm{Mt} \cdot \mathrm{a}^{-1} \text { Concentrate } \\
\text { b. } 2 \mathrm{Mt} \cdot \mathrm{a}^{-1} \text { Ilmenite/Rutile } \\
\text { c. } 390 \mathrm{kt} \cdot \mathrm{a}^{-1} \text { Zircon } \\
\text { d. } 80 \mathrm{kt} \cdot \mathrm{a}^{-1} \text { Monnzite concen- } \\
\quad \text { trate } \\
\text { e. }-\mathrm{kt} \cdot \mathrm{a}^{-1} \text { Synthetic rutile } \\
\text { III. } \\
\text { a. } 30 \mathrm{Mt} \cdot \mathrm{a}^{-1} \\
\text { b. } 40 \mathrm{kt} \cdot \mathrm{a}^{-1} \\
\text { c. } 400 \mathrm{kt} \cdot \mathrm{a}^{-1} \\
\text { d. } 20 \mathrm{kt} \cdot \mathrm{a}^{-1} \\
\text { e. }-\mathrm{kt} \cdot \mathrm{a}^{-1} \\
\text { f. }-\mathrm{kt} \cdot \mathrm{a}^{-1}\end{array}$ & $\begin{array}{l}\text { I. } 0.02-0.3,0.03-0.12 \mathrm{U} \\
\text { II. } \\
\text { a. } 0.3-3 \mathrm{Th},<0.1-0.8 \mathrm{U} \\
\text { b. } 0.2-2 \mathrm{Th},<0.1-0.6 \mathrm{U} \\
\text { c. } 0.6-1.2 \mathrm{Th}, 1-4 \mathrm{U} \\
\text { d. } 40-250 \mathrm{Th}, 6-30 \mathrm{U} \\
\text { e. }<0.2-1.5 \mathrm{Th},<0.1-0.3 \mathrm{U} \\
\text { III. } \\
\text { a. }<0.2 \mathrm{Th},<0.1 \mathrm{U} \\
\text { b. } 0.3-8 \mathrm{Th}, 0.6-2.0 \mathrm{U} \\
\text { c. } 0.8-24 \mathrm{Th}, 0.1-12 \mathrm{U} \\
\text { d. } 1-20 \mathrm{Th}, 0.1-6 \mathrm{U} \\
\text { e. }<0.2-1.5 \mathrm{Th},<0.1-0.3 \mathrm{U} \\
\text { f. } 0.1-1.2 \mathrm{Th}, 0.1-1.2 \mathrm{U}\end{array}$ & $\begin{array}{l}\text { IIIa. Landfill disposal in mined } \\
\text { out area } \\
\text { IIIb. To IIIf. Dilution with inert } \\
\text { solids, then landfill dis- } \\
\text { posal }\end{array}$ \\
\hline $\begin{array}{l}\text { Titanium pigment production } \\
\text { I. Rutile/synthetic rutile } \\
\text { II. Titanium pigment } \\
\text { III. } \\
\text { a. Neutralised slurries } \\
\text { b. Solids from effluent treat- } \\
\text { ment } \\
\text { c. Liquid effluent }\end{array}$ & $\begin{array}{l}\text { I. As above } \\
\text { II. } 185 \mathrm{kt} \cdot \mathrm{a}^{-1} \text { titanium pigment } \\
\text { III. } \\
\text { a. } 200 \mathrm{kt} \cdot \mathrm{a}^{-1} \\
\text { b. } 200 \mathrm{kt} \cdot \mathrm{a}^{-1} \\
\text { c. }-\end{array}$ & $\begin{array}{l}\text { I. As above } \\
\text { II. }<0.01 \mathrm{Th},<0.01 \mathrm{U} \\
\text { III. } \\
\text { a. } 1.2 \mathrm{Th}, 0.35 \mathrm{U} \\
\text { b. } 0.8-1.4 \mathrm{Th}, 0.3-0.5 \mathrm{U} \\
\text { c. }<0.1 \mathrm{Th},<0.1 \mathrm{U}\end{array}$ & $\begin{array}{l}\text { IIIa. Landfill disposal } \\
\text { IIIb. Landfill disposal } \\
\text { IIIc. Ocean discharge }\end{array}$ \\
\hline $\begin{array}{l}\text { Zirconium and ceramics indus- } \\
\text { try } \\
\text { I. Zircon } \\
\text { II. Zirconia, refractory materi- } \\
\quad \text { als, ceramics, glazes } \\
\text { III. } \\
\text { a. Sludge } \\
\text { b. Chlorinator residues } \\
\text { c. Dust } \\
\text { d. Slag }\end{array}$ & $\begin{array}{l}\text { I. As above } \\
\text { II. - } \\
\text { III. - }\end{array}$ & $\begin{array}{l}\text { I. As above } \\
\text { II. - } \\
\text { III. - }\end{array}$ & \\
\hline
\end{tabular}

\section{TENORM INVENTORY}

Tables 1 and 2 outline the areas of industry where NORM is handled in Australia, the scale of production, typical radionuclide concentrations, and how resulting wastes are managed.

\section{REGULATION FOR TENORM}

\section{Current regulatory requirements}

The application of radiological protection regulations is currently not uniform throughout Australia. There are nine separate jurisdictions (State, Territory and Commonwealth) having responsibility for radiation safety legislation associated with NORM/TENORM. The lack of uniformity in areas such as licensing, exemption limits and definitions (refer to
Tables 3 and 4), and the fact that some parts of regulations in individual jurisdictions do not meet current international best practice, creates problems across jurisdictions. Although safety has not been compromised, uniformity of legislation is regarded as a means of avoiding higher costs for the end user due to higher transport and production costs.

The development of acceptable and uniform national radiation protection legislation became one of the responsibilities of the Australian Radiation Protection and Nuclear Safety Agency (ARPANSA), when it was established as Australia's national radiation protection body to administer the ARPANS Act (1998) and Regulations (1999). ARPANSA also accepted the responsibility ${ }^{* 2}$ to develop a national regulatory frame-

*2 Australian Health Ministers' Conference on 4 August 1999. 
Table 1 (cont) : Summary of NORM in Australian Industries and Materials

\begin{tabular}{|c|c|c|c|}
\hline $\begin{array}{l}\text { Category of NORM } \\
\text { I. Raw material } \\
\text { II. Product(s) } \\
\text { III. Waste/by-product }\end{array}$ & $\begin{array}{l}\text { Scale of mining/production and } \\
\text { waste generation in Australia } \\
\text { (estimated) }\end{array}$ & $\begin{array}{l}\text { Typical radionuclide concentra- } \\
\text { tions }\end{array}$ & $\begin{array}{l}\text { Waste management or by-prod- } \\
\text { uct use }\end{array}$ \\
\hline $\begin{array}{l}\text { Alumina Production } \\
\text { I. Bauxite } \\
\text { II. Alumina } \\
\text { III. Red mud }\end{array}$ & $\begin{array}{l}\text { I. } \quad 55 \mathrm{Mt} \cdot \mathrm{a}^{-1} \text { bauxite } \\
\text { II. } \quad 16 \mathrm{Mt} \cdot \mathrm{a}^{-1} \text { alumina } \\
\text { III. }>20 \mathrm{Mt} \cdot \mathrm{a}^{-1} \text { red mud }\end{array}$ & $\begin{array}{ll}\text { I. } & 0.5 \mathrm{kBq} \cdot \mathrm{kg} \mathrm{Th}, 0.12 \mathrm{U}, 0.7 \\
& { }^{40} \mathrm{~K} \\
\text { II. } & \text { n.d. Th, n.d. U } \\
\text { III. } & 1.3 \mathrm{kBq} \cdot \mathrm{kg}^{-1} \mathrm{Th}, 0.4 \mathrm{U} \text {, } \\
& 0.15^{40} \mathrm{~K}\end{array}$ & III. Landspreading \\
\hline $\begin{array}{l}\text { Copper mining and processing } \\
\text { I. copper ore } \\
\text { II. Copper concentrate/refined } \\
\text { metal } \\
\text { III. } \\
\text { a. Tails from flotation } \\
\text { b. Dust from smelter } \\
\text { c. Slag from smelter }\end{array}$ & $\begin{array}{l}\text { I. } 20 \mathrm{Mt} \cdot \mathrm{a}^{-1} \\
\text { II. } \\
\text { a. } 800 \mathrm{kt} \cdot \mathrm{a}^{-1} \text { primary copper } \\
\quad \text { products } \\
\text { b. } 250 \mathrm{kt} \cdot \mathrm{a}^{-1} \text { refined copper } \\
\text { III. } \\
\text { a. }-\mathrm{kt} \cdot \mathrm{a}^{-1} \\
\text { b. }-\mathrm{kt} \cdot \mathrm{a}^{-1} \\
\text { c. }-\mathrm{kt} \cdot \mathrm{a}^{-1}\end{array}$ & $\begin{array}{l}\text { I. - } \\
\text { II. a. - } \\
\text { III. } \\
\quad \text { a. } \mathrm{Bq} \cdot \mathrm{kg}^{-1} \mathrm{Th}, \mathrm{Bq} \cdot \mathrm{kg}^{-1} \mathrm{U} \\
\quad \text { b. } \mathrm{Bq} \cdot \mathrm{kg}^{-1}{ }^{210} \mathrm{~Pb},{ }^{210} \mathrm{Po} \\
\text { c. } \mathrm{Bq} \cdot \mathrm{kg}^{-1} \mathrm{Th}, \mathrm{Bq} \cdot \mathrm{kg}^{-1} \mathrm{U}\end{array}$ & $\begin{array}{l}\text { IIIa. Disposal in tailings dam } \\
\text { with } U \text { tails }\end{array}$ \\
\hline $\begin{array}{l}\text { Tantalum/tin mining and proc- } \\
\text { essing } \\
\text { I. Tantalum ore } \\
\text { II. } \\
\text { a. Tantalum concentrate } \\
\text { b. Tin } \\
\text { III. } \\
\text { a. Tantalum tails } \\
\text { b. Tin slag }\end{array}$ & $\begin{array}{l}\text { I. } 2.5 \mathrm{Mt} \cdot \mathrm{a}^{-1} \\
\text { II. } \\
\text { a. } 2.5 \mathrm{kt} \cdot \mathrm{a}^{-1} \\
\text { b. }-\mathrm{kt} \cdot \mathrm{a}^{-1} \\
\text { III. } \\
\text { a. }-\mathrm{kt} \cdot \mathrm{a}^{-1} \\
\text { b. }-\mathrm{kt} \cdot \mathrm{a}^{-1}\end{array}$ & $\begin{array}{l}\text { I. }<10 \mathrm{~Bq} \cdot \mathrm{kg}^{-1} \mathrm{Th},<60 \mathrm{~Bq} \cdot \\
\mathrm{kg}^{-1} \mathrm{U} \\
\text { II. a. } 7.5-75 \mathrm{~Bq} \cdot \mathrm{kg}^{-1}, \mathrm{U}+\mathrm{Th} \\
\text { III. } \\
\text { a. - } \\
\text { b. - }\end{array}$ & IIIa. Landfill disposal \\
\hline $\begin{array}{l}\text { Iron Smelting } \\
\text { I. Iron ore } \\
\text { II. Iron (+steel) } \\
\text { a. Furnace slag } \\
\text { b. Dust } \\
\end{array}$ & $\begin{array}{l}\text { I. } 200 \mathrm{Mt} \cdot \mathrm{a}^{-1} \\
\text { II. } 8 \mathrm{Mt} \cdot \mathrm{a}^{-1} \\
\text { III. } \\
\text { a. }-\mathrm{Mt} \cdot \mathrm{a}^{-1} \\
\text { b. }-\mathrm{kt} \cdot \mathrm{a}^{-1}\end{array}$ & $\begin{array}{l}\text { I. - } \\
\text { III. } \\
\text { a. - } \\
\text { b. }<100 \mathrm{k} \mathrm{Bq} \cdot \mathrm{kg}^{-1} \quad{ }^{210} \mathrm{~Pb} \text { and } \\
{ }^{210} \mathrm{Po}\end{array}$ & $\begin{array}{l}\text { III. Landfill disposal of slag and } \\
\text { dust }\end{array}$ \\
\hline $\begin{array}{l}\text { Phosphate industry } \\
\text { I. phosphate rock } \\
\text { II. Fertilisers, phosphoric acid } \\
\text { III. } \\
\text { a. Phosphogypsum } \\
\text { b. Calcium fluoride } \\
\text { c. Furnace slag and dust } \\
\text { d. Scale }\end{array}$ & $\begin{array}{l}\text { I. } 2 \mathrm{Mt} \cdot \mathrm{a}^{-1} \text { (local rock) } \\
\text { II. } \\
\text { a. } 4 \mathrm{Mt} \cdot \mathrm{a}^{-1} \text { superphosphate } \\
\text { b. } 100 \mathrm{kt} \cdot \mathrm{a}^{-1} \text { acid }(<1993) \\
\text { c. ADP } \\
\text { III. } \\
\text { a. } 250 \mathrm{kt} \cdot \mathrm{a}^{-1}(<1993) \\
\text { b. } 90 \% \text { of ore } \\
\text { c. } 1 \% \text { of ore as dust and } 85 \% \\
\text { as slag }\end{array}$ & 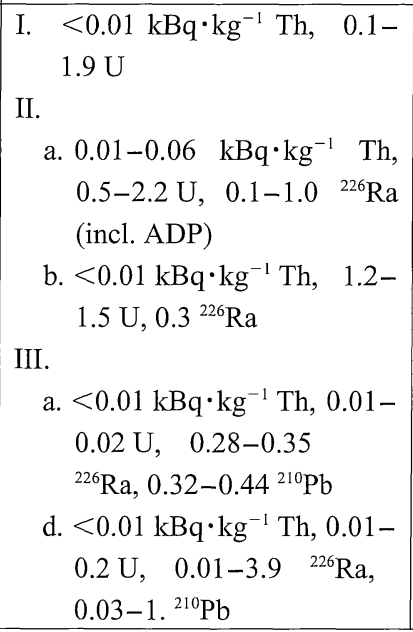 & $\begin{array}{l}\text { IIIa. Stockpiled on site /plaster- } \\
\text { board manufacture (10\%) }\end{array}$ \\
\hline
\end{tabular}

work for incorporation by individual jurisdictions. This involved the development of standards, codes and guidelines for national radiation protection and nuclear safety. The new guidelines are contained in the National Directory for Radiation Protection (the Directory). The draft edition of the Directory (Edition 1) closed to public submissions in April 2004 after significant stakeholder input, including submissions from mineral processing industries affected by NORM.

At its meeting on 27 July 2004, the Australian Health Ministers Conference (AHMC) endorsed Edition 1 of the National Directory for Radiation Protection as the uniform national framework for radiation protection in Australia. 
Table 1 (cont): Summary of NORM in Australian Industries and Materials

\begin{tabular}{|c|c|c|c|}
\hline $\begin{array}{l}\text { Category of NORM } \\
\text { I. Raw Material } \\
\text { II. Product(s) } \\
\text { III. Waste/by-product }\end{array}$ & $\begin{array}{l}\text { Scale of mining/production and } \\
\text { waste generation in Australia } \\
\text { (estimated) }\end{array}$ & $\begin{array}{l}\text { Typical radionuclide concentra- } \\
\text { tions }\end{array}$ & $\begin{array}{l}\text { Waste management or by-prod- } \\
\text { uct use }\end{array}$ \\
\hline $\begin{array}{l}\text { Oil And Gas Production } \\
\text { I. Natural oil and gas } \\
\text { II. Purified oil and gas } \\
\text { III. } \\
\text { a. Sands and sludge } \\
\text { b. Soft scales } \\
\text { c. Hard scales and film }\end{array}$ & $\begin{array}{l}\text { I. - } \\
\text { III. } \\
\quad \text { a. } 200 \mathrm{t} \\
\quad \text { b. }- \\
\text { c. } 1-2 \mathrm{t}\end{array}$ & $\begin{array}{l}\text { III. } \\
\begin{aligned} \text { a. } & <0.01 \mathrm{kBq} \cdot \mathrm{kg}^{-1} \mathrm{Th}, \quad< \\
& 0.01 \mathrm{U}, \quad 0.1-10 \quad{ }^{226} \mathrm{Ra}, \\
& 0.05-4{ }^{228} \mathrm{Ra}, 0.01-1^{210} \mathrm{~Pb} \\
\text { b. } & <0.01-0.07 \mathrm{Na} \cdot \mathrm{kg}^{-1} \mathrm{Th}, \\
& <0.01 \mathrm{U}, \quad 0.1-10{ }^{226} \mathrm{Ra}, \\
& 0.05-4{ }^{228} \mathrm{Ra}, 0.01-1^{210} \mathrm{~Pb} \\
\text { c. } & <0.01 \mathrm{~Bq} \cdot \mathrm{kg}^{-1} \mathrm{Th},<0.01- \\
& 0.5 \mathrm{U}, 0.1-100{ }^{226} \mathrm{Ra}, 0.1- \\
& 40{ }^{228} \mathrm{Ra}, 0.1-300{ }^{210} \mathrm{~Pb}\end{aligned}\end{array}$ & $\begin{array}{l}\text { IIIa. Landfill } \\
\text { Ocean discharge }\end{array}$ \\
\hline $\begin{array}{l}\text { COAL-FIRED POWER GEN- } \\
\text { ERATION } \\
\text { I. Coal } \\
\text { II. Electrical power } \\
\text { III. } \\
\quad \text { a. Fly ash } \\
\text { b. Bottom ash }\end{array}$ & $\begin{array}{l}\text { I. } \\
\text { a. } 35 \mathrm{Mt} \cdot \mathrm{a}^{-1} \text { Black coal } \\
\text { b. } 38 \mathrm{Mt} \cdot \mathrm{a}^{-1} \text { Brown coal } \\
\text { III. } \\
\text { a. } 8.6 \mathrm{Mt} \cdot \mathrm{a}^{-1} \text { Fly ash } \\
\text { b. } 1 \mathrm{Mt} \cdot \mathrm{a}^{-1} \text { Bottom ash }\end{array}$ & 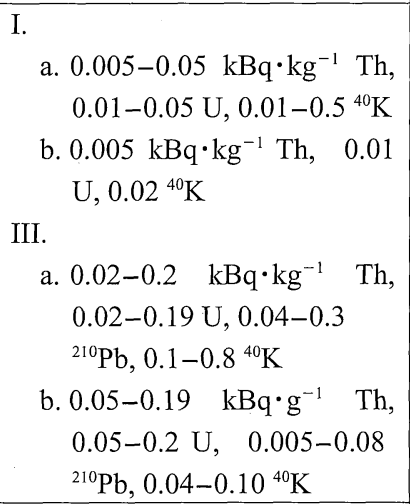 & $\begin{array}{l}\text { IIIa. Landfill disposal, cement } \\
\text { and brick manufacture } \\
\text { IIIb. Landfill disposal, road con- } \\
\text { struction }\end{array}$ \\
\hline $\begin{array}{l}\text { WATER TREATMENT } \\
\text { I. Surface or bore water } \\
\text { II. Purified potable water } \\
\text { III. } \\
\text { a. Sludge } \\
\text { b. Resins and cartridges }\end{array}$ & $\begin{array}{l}\text { I. } 1400 \mathrm{GL} \\
\text { III. } 100 \mathrm{kt} \cdot \mathrm{a}^{-1}\end{array}$ & $\begin{array}{l}\text { I. }<1 \text { Bq. }{ }^{-1}{ }^{228} \mathrm{Ra},<1 \text { Bq. } \mathrm{L}^{-1} \\
{ }^{226} \mathrm{Ra} \\
\text { II. - } \\
\text { III. - }\end{array}$ & $\begin{array}{l}\text { IIIa. Landfill disposal or land- } \\
\text { spreading } \\
\text { IIIb. Landfill disposal }\end{array}$ \\
\hline $\begin{array}{l}\text { BUILDING MATERIALS } \\
\text { I. } \\
\text { a. Raw materials } \\
\text { b. By-product wastes } \\
\text { II. Bricks, cement, plaster- } \\
\text { board, ceramic tiles }\end{array}$ & $\begin{array}{l}\text { I. b. } 15 \% \text { Phosphogypsum re- } \\
\text { cycled, } 10 \% \text { Fly ash } \\
\text { II. - }\end{array}$ & $\begin{array}{l}\text { I. b. As above } \\
\text { II. Concrete: } 0.001-0.24 \mathrm{kBq} \cdot \\
\mathrm{kg}^{-1} \mathrm{Th}, \quad 0.001-0.25^{226} \mathrm{Ra} \text {, } \\
0.005-1.5^{40} \mathrm{~K} \\
\text { Bricks: } \quad 0.001-0.2 \quad \mathrm{kBq} \cdot \\
\mathrm{kg}^{-1} \quad \mathrm{Th}, \quad 0.01-2.2{ }^{226} \mathrm{Ra} \text {, } \\
0.01-1.6^{40} \mathrm{~K} \\
\text { Plasterboard: } \quad<0.01-0.05 \\
\mathrm{kBq} \cdot \mathrm{kg}^{-1} \mathrm{Th},<0.01-0.7 \\
{ }^{226} \mathrm{Ra}, 0.025-0.1{ }^{40} \mathrm{~K} \\
\text { Ceramics: } \quad 0.02-0.2 \mathrm{kBq} \cdot \\
\mathrm{kg}^{-1} \quad \mathrm{Th}, \quad 0.03-0.2{ }^{226} \mathrm{Ra} \text {, } \\
0.16-1.4^{40} \mathrm{~K}\end{array}$ & \\
\hline
\end{tabular}

Note: - information not currently availabe to author

\section{Industry Guides}

There are a number of guidelines on NORM management within specific industries. For example, Smith $(1992)^{* 3}$ has reported on NORM in the USA petroleum industry, and Aus-

*3 As cited in Radiation Health \& Safety Advisory Council NaturallyOccurring Radioactive Material (NORM) in Australia: Issues for Discussion. 30 June 2004 tralian Petroleum Production and Exploration Association Limited (APPEA) has published guidelines for the oil industry*4. The APPEA Guideline provides guidance on NORM monitoring, management of occupational radiation exposures

${ }^{* 4}$ APPEA, 2002, as cited in Radiation Health \& Safety Advisory Council Naturally-Occurring Radioactive Material (NORM) in Australia: Issues for Discussion. 30 June 2004 
Table 2 Summary of NORM materials in terms of quantities and activities.

\begin{tabular}{|c|c|c|c|}
\hline \multirow{2}{*}{$\begin{array}{l}\text { Radionuclide content } \\
\qquad\left(\mathrm{Bq} \cdot \mathrm{kg}^{-1}\right)\end{array}$} & \multicolumn{3}{|c|}{ Quantity produced annually } \\
\hline & Small $(<1 \mathrm{kt})$ & Moderate $(1-100 \mathrm{kt})$ & Large $(>100 \mathrm{kt})$ \\
\hline Low $(<1,000)$ & & $\begin{array}{l}\text { Synthetic rutile } \\
\text { Ceramics } \\
\text { Sand blasting materials }\end{array}$ & $\begin{array}{l}\text { Alumina waste (red mud) } \\
\text { Coal ash } \\
\text { Furnace and metal smelter slags } \\
\text { Phosphogypsum } \\
\text { Phosphate fertilisers } \\
\text { Water treatment sludges } \\
\text { Tantalum tails } \\
\text { Copper tailings }\end{array}$ \\
\hline $\begin{array}{l}\text { Medium } \\
(1,000-20,000)\end{array}$ & Oil sand/sludge & Tantalum products & $\begin{array}{l}\text { Phosphate rock } \\
\text { Zircon } \\
\text { Ilmenite }\end{array}$ \\
\hline High $(>20,000)$ & $\begin{array}{l}\text { Zircon dusts } \\
\text { Copper smelter dusts } \\
\text { Oil scales } \\
\text { Iron sinter dusts }\end{array}$ & $\begin{array}{l}\text { Monazite concentrates } \\
\text { Monazite tailings }\end{array}$ & \\
\hline
\end{tabular}

and decision-making regarding NORM waste disposal. APPEA recommended a similar approach to that used in the

Table 3 Comparison of total-activity exemption levels.

\begin{tabular}{|l|c|c|c|c|c|c|c|c|c|c|c|}
\hline \multicolumn{1}{|c|}{ Nuclide } & Half -life & IAEA BSS & \multicolumn{7}{|c|}{ Australian jurisdiction } \\
\hline & & Total & ACT & ARPANSA & NSW & NT & QLD & SA & TAS* & VIC & WA \\
\hline & & MBq & MBq & MBq & MBq & MBq & MBq & MBq & MBq & MBq & MBq \\
\hline${ }^{40} \mathrm{~K}$ & $1.28 \times 10^{9} \mathrm{y}$ & 1 & & 1 & & & 1 & & & & \\
\hline $\begin{array}{l}\text { Th-nat } \\
\text { (incl. }{ }^{232} \mathrm{Th} \text { ) }\end{array}$ & $1.4 \times 10^{10} \mathrm{y}$ & 0.001 & 4 & 0.001 & 40 & & 0.001 & 5 & & 4 & 4 \\
\hline${ }^{232} \mathrm{Th}$ series & & & & & & & & & & & \\
\hline${ }^{232} \mathrm{Th}$ & $1.4 \times 10^{10} \mathrm{y}$ & & 4 & & 40 & & & 5 & & \\
\hline${ }^{228} \mathrm{Ra}$ & $5.75 \mathrm{y}$ & 0.1 & 0.004 & 0.1 & 0.04 & & 0.1 & 0.005 & & & \\
\hline${ }^{228} \mathrm{Ac}$ & $6.13 \mathrm{~h}$ & 1 & 0.04 & 1 & 0.4 & & 1 & 0.05 & & & \\
\hline${ }^{228} \mathrm{Th}$ & $1.91 \mathrm{~d}$ & 0.01 & 0.004 & 0.01 & 0.04 & & 0.01 & 0.005 & & & \\
\hline${ }^{224} \mathrm{Ra}$ & $3.66 \mathrm{~d}$ & 0.1 & 0.04 & 0.1 & 0.4 & & 0.1 & 0.05 & & & \\
\hline${ }^{220} \mathrm{Rn}$ & $55.6 \mathrm{~s}$ & 10 & & 10 & 4 & & 10 & 0.5 & & & \\
\hline${ }^{212} \mathrm{~Pb}$ & $10.6 \mathrm{~h}$ & 0.1 & 0.04 & 0.1 & 0.4 & & 0.1 & 0.05 & & & \\
\hline${ }^{212} \mathrm{Bi}$ & $60.55 \mathrm{~m}$ & 0.1 & 0.4 & 0.1 & 4 & & 0.1 & 0.5 & & & \\
\hline $\mathrm{U}-\mathrm{nat}$ & & 0.001 & 4 & 0.001 & 40 & & 0.001 & 5 & & 4 & 4 \\
\hline${ }^{238} \mathrm{U} \mathrm{series}$ & & & & & & & & & & \\
\hline${ }^{238} \mathrm{U}$ & $4.47 \times 10^{9} \mathrm{y}$ & 0.01 & 4 & 0.01 & 40 & & 0.01 & 5 & & \\
\hline${ }^{234} \mathrm{Th}$ & $24.1 \mathrm{~d}$ & 0.1 & 0.04 & 0.1 & 0.4 & 3.7 & 0.1 & 0.05 & & \\
\hline${ }^{234} \mathrm{U}$ & $2.44 \times 10^{5} \mathrm{y}$ & 0.01 & 0.004 & 0.01 & 0.04 & & 0.01 & 0.005 & & \\
\hline${ }^{230} \mathrm{Th}$ & $7.70 \times 10^{4} \mathrm{y}$ & 0.01 & 0.004 & 0.01 & 0.04 & & 0.01 & 0.005 & & \\
\hline${ }^{226} \mathrm{Ra}$ & $1.60 \times 10^{3} \mathrm{y}$ & 0.01 & 0.004 & 0.01 & 0.04 & 0.037 & 0.01 & 0.005 & & 0.0004 & 0.0004 \\
\hline${ }^{222} \mathrm{Rn}$ & $3.8235 \mathrm{~d}$ & 100 & 0.4 & 100 & 4 & & 100 & 0.5 & & \\
\hline${ }^{210} \mathrm{~Pb}$ & $22.3 \mathrm{y}$ & 0.01 & 0.004 & 0.01 & 0.04 & 0.037 & 0.01 & 0.005 & & \\
\hline${ }^{210} \mathrm{Bi}$ & $5.01 \mathrm{~d}$ & 1 & 0.04 & 1 & 0.4 & & 1 & 0.05 & & 0.04 & 0.04 \\
\hline${ }^{210} \mathrm{Po}$ & $138 \mathrm{~d}$ & 0.01 & 0.004 & 0.01 & 0.04 & 0.037 & 0.01 & 0.005 & & 0.004 & 0.004 \\
\hline
\end{tabular}

${ }^{*}$ The Tasmanian regulations exempt a natural material with concentration less than $31 \mathrm{~Bq} / \mathrm{g}$, but do not have an activity limit. Exemptions for individual radionuclides are based on 1/2,000th of the most restrictive Annual Limit on Intake (ALI) for that radionuclide. As ALI are based on ingestion/inhalation pathways only, the limits are very restrictive when compared with systems that take into account a range of exposure scenarios. 
Table 4 Comparison of activity-concentration exemption levels: current and proposed. ${ }^{3)}$

\begin{tabular}{|c|c|}
\hline State or territory & Exemption requirements \\
\hline \multicolumn{2}{|l|}{ Current } \\
\hline Queensland & BSS exemption levels on individual radionuclides \\
\hline Western Australia & Total activity concentration $30 \mathrm{~Bq} \cdot \mathrm{g}^{-1}$ \\
\hline Victoria & Total activity concentration $30 \mathrm{~Bq} \cdot \mathrm{g}^{-1}$ \\
\hline Tasmania & Total activity concentration $31 \mathrm{~Bq} \cdot \mathrm{g}^{-1}$ \\
\hline South Australia & Total activity concentration $35 \mathrm{~Bq} \cdot \mathrm{g}^{-1}$ \\
\hline \multirow[t]{2}{*}{ New South Wales* } & Total activity concentration $100 \mathrm{~Bq} \cdot \mathrm{g}^{-1}$ \\
\hline & AND if $\left[\frac{A_{1}}{40}+\frac{A_{2}}{400}+\frac{A_{3}}{4,000}+\frac{A_{4}}{40,000}\right] \geq 1 * *$ \\
\hline Northern Territory & Total activity $370 \mathrm{kBq}$ \\
\hline \multicolumn{2}{|l|}{ Proposed } \\
\hline National Directory for Radiation Protection & BSS $^{* * *}$ exemption levels on individual radionuclides \\
\hline \multicolumn{2}{|c|}{$\begin{array}{l}\text { * In NSW a radioactive ore is defined as (a) in the case of an ore that contains uranium but not thorium, } 0.02 \text { per cent by weight of ura- } \\
\text { nium; or (b) in the case of an ore that contains thorium but not uranium, } 0.05 \text { per cent by weight of thorium; or (c) in the case of an } \\
\text { ore that contains both uranium and thorium, a percentage by weight of uranium and thorium such that the expression: } \\
{[\mathrm{U} / 0.02+\mathrm{Th} / 0.05] \geq 1 \text {. }}\end{array}$} \\
\hline \multicolumn{2}{|c|}{ ** $A 1-A 4$ are activities in $\mathrm{kBq}$ for different groups of radionuclides in the NSW Regulations. } \\
\hline
\end{tabular}

uranium mining and milling and heavy mineral sands industries.

\section{OPTIONS FOR ESTABLISHING NORM MANAGEMENT CRITERIA}

\section{a) Activity concentrations}

The simplest approach is to use activity concentration as the basis for any required action. However, this may not be satisfactory due to the wide variations in the amounts of NORM to which workers, the public and the environment can be exposed in different situations involving the same material.

\section{b) Application of ALARA}

Establishing activity limits may be less effective than optimisation when dealing with NORM. This is because natural background levels vary over time and place, and because NORM concentrations in existing products, commodities and wastes can vary considerably. Optimisation, which involves application of the ALARA principle (As-Low-As-Reasonably-Achievable), requires consideration of a range of factors, including social and economic impacts, of any NORM management strategy.

\section{c) Risk-based assessment}

In principle the risk-based assessment approach is the most desirable, but the wide range of situations in which exposures to NORM can occur make it difficult to develop a single, standard approach. In many of these situations, particularly where relatively small quantities of NORM are involved, it is possible to make an assessment of potential risks by analogy with the natural background occurrence of NORM (for example, fertiliser spreading, radon exhalation and/or emission of gamma radiation from granite slabs or clay bricks). Estimation of NORM dose/risk as a fraction or multiple of the natural background dose/risk is another possible approach. The process of risk assessment depends on the hazard and can be simple or detailed.

\section{CASE STUDY RELATED TO TENORM}

A Task Group from Japan visited Australia between 10-14 February 2003. The group included Prof. Toshiso KosAKO (Japan Project Leader, RWM, Tokyo University), Prof. Takao IIDA, Nagoya University; Dr, Hirokuni YAMANISI, National Institute of Fission Science, Dr. Takeshi Iімото, Tokyo University and Dr. Nobuyiki SUGIURA, Tokyo University.

Preliminary discussions were held with a number of technical experts in Australia including representatives from ANSTO, the New South Wales Environment Protection Agency and followed up with a visit to the large mining operation site at the Roxby Downs, Olympic Dam Project in South Australia

Issues discussed included:

- an update on NORM in the Mining Industry which addressed recent ANSTO involvement with the IAEA on NORM, the implementation of BSS 115 since 1996 and a review of the Draft Safety Standard 161"Radionuclide content in commodities not requiring regulation for purposes of radiation protection",

- a summary of NORM issues relevant to the Australian Mining Industry,

- an overview of the radon issues in TENORM,

- international regulations existing in the NORM industry and wastes,

- disposal of radioactive material,

- exemption levels for NORM wastes,

- waste issues arising from uranium mining,

- the use of phosphate rock in fertiliser production and consumer goods,

- radon in terms of Technology Enhanced Natural Radiation.

Discussions with the EPA focussed on rehabilitation of 

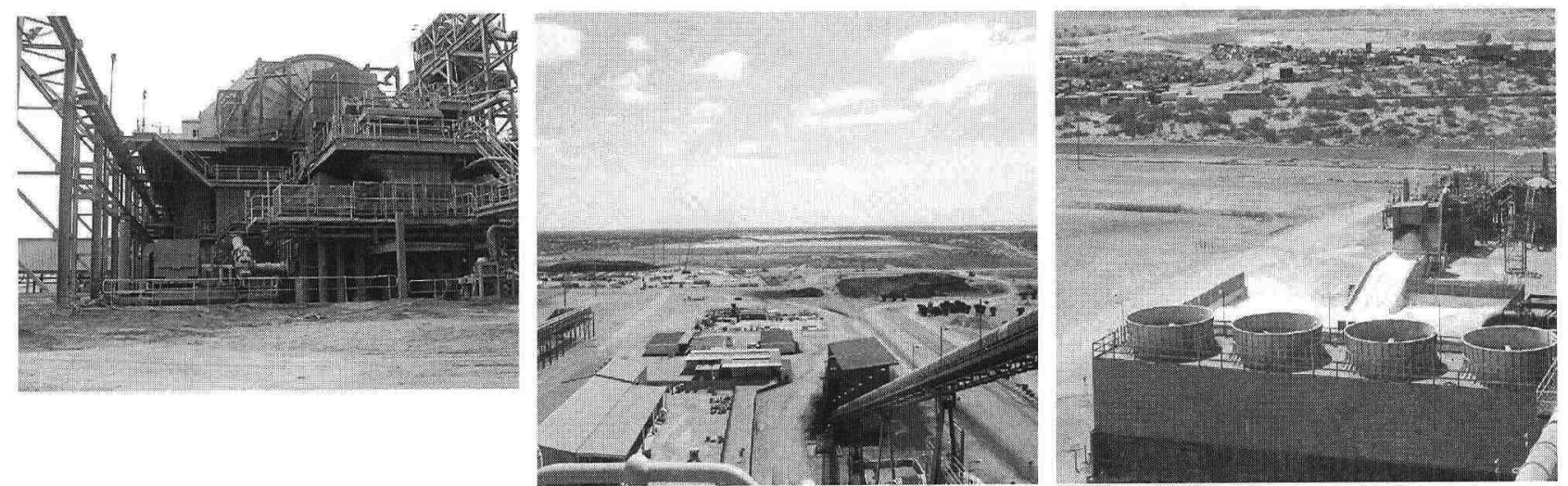

Fig.1 Olympic Dam Project site, Roxby Downs, South Australia.

mineral sand mining sites, rehabilitation of an old radium refinery, assessments of ${ }^{210} \mathrm{Po}$ and ${ }^{210} \mathrm{~Pb}$ in smelter/sinter plant dusts, occurrences in refractory bricks, ceramic insulators, glazed products, zircon abrasives and radionuclides in coal washery scales. Also discussed was the need to approve and formalise disposal and recycle options and the need to decide on regulatory requirements for the higher-activity scales and sludges.

A visit to the Roxby Downs, Olympic Dam Project site was made on Thursday 13th February. The site visit provided an insight into operational control issues relating to NORM on a large mining site, the regulatory controls that are required and the deportment of the radionuclides in smelter dusts. Figure 1 provide an oversight of the magnitude of the Olympic Dam Operations site.

\section{PROBLEMS TO BE SOLVED}

The major issues for managing NORM/TENORM waste in Australia (and worldwide) include:

\section{a) Accepted guidelines}

As previously mentioned, the application of radiological protection regulations is currently not uniform throughout Australia. There are nine separate jurisdictions (State, Territory and Commonwealth) having responsibility for radiation safety legislation associated with NORM and TENORM. In some jurisdictions, there are additional exemption provisions, such limits on activity concentration or definitions of 'mineral substances.'

The lack of uniformity in areas such as licensing, exemption limits and definitions, and the fact that some parts of regulations in individual jurisdictions do not meet current international best practice, creates problems across jurisdictions, such as when transporting NORM/TENORM commodities across national or jurisdictional boundaries. Although safety has not been compromised, uniformity of legislation is regarded as a means of avoiding higher costs for the end user due to higher transport and production costs.

These inconsistencies are an important matter, and will be addressed via the National Directory for Radiation Protection, which is being developed by the Australian Radiation Protection and Nuclear Safety Agency (ARPANSA).

\section{b) Methods of disposal}

There is no clear national or international agreement on acceptable methods of disposal of NORM waste. There are various options for disposal of NORM waste, each of which has advantages and disadvantages.

\section{c) Measurement}

Pipe geometry can affect the capacity of the oil and gas industry to assess whether the level of radioactivity in scale inside pipes, pumps, valves and other equipment requires remedial action.

\section{d) Environment}

The effects of ionizing radiation on non-human species have only recently received attention from the scientific community. The traditional approach was to assume that if humans were protected then all other species were automatically protected. As a first step in evaluating the effects of ionizing radiation on other species, the International Commission on Radiological Protection (ICRP) has published guidelines on assessing these effects. ${ }^{* 5}$

\section{e) Occupational health}

Management of materials containing NORM can lead to occupational health issues. In some industries these issues are already being addressed; for example, in the oil and gas industry radiation protection measures are implemented for staff undertaking maintenance on NORM contaminated equipment. In industries where NORM has not been recognised as a potential issue, occupational health matters may not be adequately addressed.

\section{f) Public health}

The use of products containing NORM, or the disposal of NORM bearing wastes may give rise to public health issues. For example, there is potential for issues to arise in industries where awareness is low and no NORM management procedures are implemented. With some NORM-containing products, such as thorium gas mantles, warnings must be provided to reduce the potential for inhalation of the fine dust that can

\footnotetext{
*5 International Commission on Radiological Protection, 2002, as cited in Radiation Health \& Safety Advisory Council NaturallyOccurring Radioactive Material (NORM) in Australia: Issues for Discussion. 30 June 2004
} 
result from damaged mantles, and manufacturers are encouraged to produce non-radioactive alternatives, where possible.

\section{g) Contaminated sites}

In 1985 the National Health and Medical Research Council (NHMRC) published a statement "Guidelines for Remedial Action in Areas where Residues from Mineral Sand Mining and Processing have been Deposited. ${ }^{* * 6}$ Since that time radiation protection standards have changed considerably.

There has been very little published in Australia on criteria for clean up of sites contaminated with radioactive material. Internationally, the IAEA has published some documents on this subject and others are in preparation. The ICRP has also published recommendations on the disposal of long-lived solid radioactive waste, which are relevant to remediation of contaminated sites. It is now considered that there may be a requirement for the development of appropriate guidance on remediation criteria for sites contaminated with radioactive materials, including review of the 1985 NHMRC statement. This should take account of the IAEA, ICRP and other relevant guidelines.

\section{CONCLUSIONS}

The issue of NORM and TENORM is a recurring one, and will be ever-present as long as mined materials such as coal, bauxite, ores and the like, which contain NORM at background levels, are processed in large quantities.

In Australia's case, although regulation of NORM and TENORM is not consistent across its nine jurisdictions, each set of regulations is somewhat similar and safety has not been compromised. However, replacement of these with a uniform regulatory framework for radiation protection would alleviate problems with transportation of the material within the whole of Australia. It is also noteworthy that the regulations in some jurisdictions do not completely meet current international best practice, whereas the proposed national framework would indeed do so.

For improved occupational and public health, NORM/TENORM must be recognised as an important issue in industry, and warnings should be issued where relevant. Ideally, there should also be an international agreement and guidelines concerning measurement and method of disposal of the material, taking into account specific environmental concerns.

Most of these objectives are currently underway.

\section{REFERENCES}

1) Radiation Health \& Safety Advisory Council: NaturallyOccurring Radioactive Material (NORM) in Australia: Issues for Discussion, 30 June 2004, (2004).

2) M. B. Cooper: Naturally occurring radioactive materials (NORM) in Australian industries - Review of current inventories and future generation. (2003).

3) R. Secomb, D. Collier: National uniformity for radioactive protection in Australia and the implications in regard to NORM, FNCA Workshop on Radioactive Waste Management Kuala Lumpur, 27 September-1 October 2004, (2004).

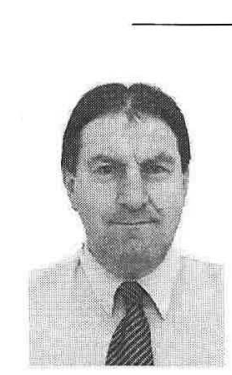

\section{Lubi Dimitrovski}

Head, Waste Operations \& Technology Development, Safety and Radiation Science, Australian Nuclear Science \& Technology Organisation (ANSTO).

*" NHMRC, 1985, as cited in Radiation Health \& Safety Advisory Council Naturally-Occurring Radioactive Material (NORM) in Australia: Issues for Discussion. 30 June 2004 